\title{
Recent Advancements in Cytotoxic T Lymphocyte Generation Methods Using Carbohydrate-Coated Liposomes
}

\author{
Yuzuru Ikehara, Masahiro Yamanaka, and Takashi Yamaguchi \\ Molecular Medicine Team of Research Center for Medical Glycoscience, National Institute of Advanced Industrial Science and \\ Technology (AIST), Tsukuba, Ibaraki 305-8568, Japan \\ Correspondence should be addressed to Yuzuru Ikehara, yuzuru-ikehara@aist.go.jp
}

Received 29 January 2010; Accepted 22 April 2010

Academic Editor: Hanchun Yang

Copyright (๑) 2010 Yuzuru Ikehara et al. This is an open access article distributed under the Creative Commons Attribution License, which permits unrestricted use, distribution, and reproduction in any medium, provided the original work is properly cited.

Both tumor-specific $\mathrm{CD}^{+}$and $\mathrm{CD}^{+}{ }^{+}$cells have been identified, and the latter is known as a major effector of adaptive antitumor immune responses. Optimal antitumor immune responses are considered to require the concomitant activation of both $\mathrm{CD} 8^{+}$and $\mathrm{CD}^{+} \mathrm{T}$ cells and the additional selective activation of $\mathrm{CD} 4^{+} \mathrm{T}$ cells with helper, but not regulatory function. As optimal antitumor immune responses are generated by the concomitant activation of both $\mathrm{T}$ cell types, it is necessary for vaccine methods involving cytotoxic T-lymphocytes (CTLs) generation to possess a mechanism whereby antigen presenting cells can present administrated exogenous antigens on not only Major histocompatibility complex (MHC) class II, but also MHC class I molecules.

\section{Introduction}

We have previously reported the development of a new drug delivery system (DDS) based on the carbohydrate recognition properties of phagocytic cells to control metastatic cancer in extranodal lymphoid tissue of the abdominal cavity [1]. Further, we demonstrated that our DDS could be used for the induction of CTLs through the presentation of exogenous antigens on MHC class I molecules of phagocytic cells [2].

In accordance with findings from many attempts, including ours, to generate antigen-specific CTLs, this paper provides an overview of current trials of liposome-based vaccines. Furthermore, we discuss the feasibility concerning our vaccination technique by summarizing accumulated knowledge regarding receptor candidates.

\section{Overview}

In order to reject invading pathogens and cancer cells, expansion of $\mathrm{T}$ cells is known to be activated by small peptides on Major histocompatibility complex (MHC) class
I or MHC class II molecules on the cell surface of antigenpresenting cells (APCs) such as dendritic cells (DCs) and macrophages. We will mainly introduce recent progression of vaccine methods to generate $\mathrm{CD} 8^{+}$cytotoxic $\mathrm{T}$ lymphocytes (CTLs) in this paper, while first mentioning the indispensable roles of $\mathrm{CD} 4^{+}$helper $\mathrm{T}$ cells that support the expansion and persistence of CTLs [3-5]. Indeed, optimal antitumor immune responses are generated by the concomitant activation of both $\mathrm{CD}^{+}$and $\mathrm{CD} 4^{+} \mathrm{T}$ cells because of the selective activation of $\mathrm{CD} 4^{+} \mathrm{T}$ cells with helper, but not regulatory functions [6]. Generally, exogenous antigens presented by MHC class II molecules are intended for $\mathrm{CD}^{+} \mathrm{T}$ cells, whereas internal antigens from the cell itself, components of virus infected cells, and cancer antigens are presented on $\mathrm{MHC}$ class I molecules for activation and expansion of $\mathrm{CD} 8^{+}$ $\mathrm{T}$ cells. Consequently, it is necessary for vaccine development methods involving CTL generation to possess a mechanism whereby administrated exogenous antigens can be presented not only on MHC class II, but also class I molecules of APCs [6-8].

For vaccine development methods whereby exogenous antigens are exhibited both on MHC class I and class II molecules to induce antigen-specific $\mathrm{CD} 8^{+}$and $\mathrm{CD} 4^{+} \mathrm{T}$ cells, 
our novel drug delivery system (DDS) using oligomannosecoated liposomes (OMLs) [1] that target phagocytic cells can be tailored for this purpose [2]. Indeed, a novel OMLbased vaccine could reject transplanted tumor cells, prevent progression of encephalitis and vertical transmission, and reduce offspring mortality of Neospora caninum as shown in a feasibility study for its clinical use [2, 9-11].

OML-based vaccines produce strong adjuvanticity for CTLs. As liposomes coated by oligomannose are exclusively taken up by $\mathrm{F} 4 / 80^{+}$intraperitoneal mononuclear cells and gathered at extranodal lymphoid tissues, the so-called "milky spots in abdominal cavity" [1], the underlining mechanisms of OML-based vaccine appear to be accompanied by an immune surveillance system for detecting pathogens invading the abdominal cavity in either a mannose dependant or mannose independent manner. Important roles for macrophages and complement systems are well known in the clearance of foreign materials, invading bacteria, and tumor cells from the abdominal cavity. Moreover, it is the milky spots that are the exact locus of this clearance process [1215]. Taken together, a line of clearance process for OMLs may associate with strong adjuvanticity to induce CTLs.

Some diseases such as hepatitis $\mathrm{C}$ virus infection and malignancies still remain to have vaccine methods developed for them using disease-specific CTLs by elucidating their basic roles $[5,16,17]$. Many attempts to generate antigen-specific CTLs have been conducted, based on new experimental evidence. In accordance with these efforts, this paper will provide an overview of current trials concerning liposome-based vaccine delivery, and we discuss the feasibility of an OML-based vaccine based on recently accumulated knowledge of the carbohydrate recognition system as a target for OML-based vaccine delivery systems.

\subsection{Liposome-Based Vaccine Delivery to Generate CTLs.} Based on results from materials investigated for immunization, many types of liposomes have been tested for use in attempts to increase the effect of CTL generation against delivered vaccine antigens. New materials used in liposome preparation have been investigated to see whether they could effectively generate CTLs while monitoring the following three effects [18-20]: (1) an increase in the fusion efficiency between the cell membrane and liposomes, (2) the stabilization of liposomes in blood circulation, and (3) efficient delivery of vaccine antigens to APCs.

The approach tried first was to increase the fusion efficiency between the cell membrane and liposomes, because external antigens spilling from endosomes into the cytosol is considered to be the most important step for entry into the class I processing pathway for CTL generation $[18,21,22]$. To this purpose, use of peptide sequences referred to as antennapedia homeodomain [23, 24], and the hemolytic protein of Listeria monocytogenes, listeriolysin $\mathrm{O}$ [25], succeeded in enhancing the introduction of CTL epitopes into the class I processing pathway, resulting in the increased generation of CTLs. Furthermore, fusogenic liposomes prepared by fusing simple liposomes with Sendai virus particles can deliver encased antigens into the cytosol to generate CTLs [26].
Retaining liposomes in blood circulation is another way to increase the efficiency of CTL generation. Increasing retention time has been achieved by reducing surfacemacromolecule interaction, which provides less opportunity for liposomes uptake by phagocytic cells and hepatocytes $[27,28]$. The approach is highly effective for induction of CTLs against antigens encased in liposomes [29, 30]. To this purpose, polyethylene glycol (PEG)-modified lipids have become universally used in the preparation of liposomes (PEG-liposome) [29-31], which can more greatly enhance the generation of a CD8 ${ }^{+} \mathrm{T}$ cell response than when given in soluble form or in conventional or positively charged liposomes [32]. Moreover, new lipids isolated from Archaea have also been used in the preparation of liposomes because of their stabilizing effect on liposomes in a manner similar to that for PEG-modified lipids. Archaea liposomes showed higher stability against extreme $\mathrm{pH}$, oxidation, elevated temperatures, and action of lipases than conventional liposomes [33-35].

While higher stabilization in blood circulation increases CTL generation as discussed above, enhanced uptake by phagocytic cells has been indicated to elicit strong adjuvanticity to induce antigen-specific CTL responses [19]. The relevant examples of specific delivery of liposomes to phagocytic cells are cationic liposomes and OMLs. Positive charge on a liposome surface enhances uptake by APCs more than neutral membranes, and more robust immune responses for CTL generation and antibody production were seen in mice immunized using positively charged liposomes than with neutral liposomes [23, 28, 36-38]. To add positive charge to the surface of liposomes in earlier studies, cationic cholesterol derivatives such as $3 \beta\left[\mathrm{N}-\left(\mathrm{N}^{\prime}, \mathrm{N}^{\prime}\right.\right.$ dimethylaminoethane)-carbamyl] cholesterol hydrochloride were frequently used.

Coating with ligands for pattern recognition receptors such as a mannose receptor (MR) on APCs is expected to have the analogous effect of adding positive charge to the liposome surface using cationic cholesterol derivatives, because ligand binding triggers endocytosis of liposomes by APCs such as DCs and macrophages [39, 40]. Related to this concept, Chikh et al. have indicated a line of phagocytic receptors for a variety of ligands, FC $\gamma$ RI [41, 42], mannose $[39,40], \alpha \mathrm{M} \beta 2$ integrin (CD11b CD18) [43, 44], CD36, and $\alpha \mathrm{v} \beta 5$ integrin [45], for forced uptake by APCs [19].

\subsection{Carbohydrate Coatings on Liposomes to Deliver Vaccine} Antigen to APCs. To induce robust immune responses using carbohydrates recognition by phagocytic cells, either mannose residues coupling on antigens or coating on an antigen encased liposomes appears to show promise. Using these methods, antigens were able to efficiently deliver phagocytic cells such as APCs, due to the uptake by mannose recognition receptors such as macrophage mannose receptor (MMR, CD206) and DC-specific intercellular adhesion molecule (ICAM)-3-grabbing nonintegrin (DC-SIGN) preferentially expressed on them, resulting in effective induction of CTLs $[1,46-50]$. It may be associated with a nature of mannose residues, which significantly enhances immunogenicity of antigens and strongly promotes DC maturation through 
TLR4 function [51]. Concerning carbohydrate coupling on antigen, not only the high-mannose oligosaccharide [47] and O-linked short mannose (2-3 mannoses) from $P$. pastoris [48-50], but also fucosylated oligosaccharides such as Lewis $\mathrm{X}$ or Lewis B [50], could be used to specifically deliver to dendritic cell by the other preferential binding specificity of DC-SIGN. Indeed, either mannosylated or fucosylated antigens could enhance CTLs responses depending on antigen presentation via class I molecules. $[47,49,50]$.

Concerning mannose residues coating on liposome surfaces, we used mannotriose-dipalmitoylphosphatidylethanolamine (Man3-DPPE) [52] in the preparation of liposomes with dipalmitoyl phosphatidylcholine and cholesterol, at a molar ratio of $1: 10: 10[52,53]$. Man3-DPPE, a neoglycolipid that is composed of Man3 and DPPE, is synthesized by reductive amination between an aldehyde group of oligosaccharides and the amino group of DPPE [52]. Because of the hydrophobic lipid moiety of the neoglycolipid, Man3-DPPE can easily be incorporated into the lipid bilayer of liposomes. The liposomes contain Man3DPPE, and we have named them OMLs.

As mentioned in our previous studies, OMLs injected into the abdominal cavity are taken up by $\mathrm{CD} 11 \mathrm{~b}^{+}$phagocytic cells that deliver material to milky spots $[1,46]$. Indeed, when 5-fluorouracil (5-FU) was encapsulated in the OMLs, more than $60 \%$ of the administered 5-FU accumulated in the omentum where milky spots gathered [1]. In other words, the OML-based DDS targets CD11b ${ }^{+}$phagocytic cells that act as cellular vehicles for material delivery. Recent use of hematopoietic or mesenchymal stem cells as cellular vehicles has led to significant progress in gene delivery techniques, while Burke has indicated the advantages of using phagocytic cells as natural cellular vehicles [54]. Phagocytic cells such as macrophages in the abdominal cavity take up large amounts of particles and accumulate them at not only lymphoid tissue, but also various pathological sites such as cancer lesions, wounds, atherosclerotic plaques, and arthritic joints [54]. Consequently, for delivery of materials, OMLs are a valuable tool for exploiting the nature of phagocytic cells.

We recently demonstrated the usefulness of OMLs as carriers for the delivery of vaccine antigen to generate and expand CTLs by employing ovalbumin (OVA) as a model cancer antigen [2]. Indeed, APC came to express OVAderived peptides obtained by OML-based delivery in the context of both MHC class I and II molecules, which were evaluated by the detection of interferon gamma (IFN $\gamma$ ) production in the coculture with OML-delivered APCs and either $\mathrm{CD} 8^{+}$or $\mathrm{CD} 4^{+} \mathrm{T}$ cells from the spleens of $\mathrm{T}$ cell receptor transgenic mice OT-I (specific for $\mathrm{H}-2 \mathrm{~Kb} / \mathrm{OVA}_{257-264}$ ) $[55,56]$ or OT-II (H-2Ab/OVA $323-339)$ [57], respectively [2]. Moreover, only the spleen cells from mice immunized with OML-OVA, but not bare liposomes without coating-encased OVA, showed cytotoxicity against E.G7-OVA, and only the mice preimmunized with subcutaneous challenge by OMLOVA rejected E.G7-OVA, but not EL4. These results together indicate that the OMLs can be used as an effective antigen delivery system for immunotherapy activating both CTL and Th subsets. OMLs are very useful not only for the promotion of nonglycosylated protein uptake by APCs, but also for the enhancement of antigen processing of encased antigens. This advantage of OML-mediated immunization will hopefully facilitate the simultaneous activation of tumor antigen-specific $\mathrm{CD}^{+}$and $\mathrm{CD} 8^{+} \mathrm{T}$ cells [2], and have the potential for use in cancer immune therapy [9].

It is well known that carbohydrates binding protein on APCs and complement lectin pathways recognize conserved motifs of glycans on pathogens. Carbohydrates binding proteins on phagocytic cells participate in the capture of materials to internalize, while the complement lectin pathway actively generates peptide fragments from C3, facilitating opsonophagocytosis by phagocytic cells through the complement receptors (CRs). Inhibition of complement component $\mathrm{C} 3$ and complement receptor type 3 (CR3, CD11b/CD18) could block the uptake process of OMLs by phagocytic cells $[58,59]$. These observations support the hypothesis that carbohydrates binding receptors promote the uptake of liposomes in accordance with the activated lectin pathway, acting as an essential step in robust CTL responses against antigens encased in liposomes [59-61].

\section{Possible Target Receptors on APCs Using Liposomes with Carbohydrate Coatings}

In this section, we focus on receptor candidates on APCs for uptake of liposomes with carbohydrate coatings. Based on recent advancements in technologies to investigate structure-function relationships of glycans, knowledge about the properties of carbohydrate-binding proteins has dramatically increased, and offers their possible use as targets for the delivery of vaccine antigens. Here, we introduce DCSIGN (CD209), MMR (CD206), and CRs to illustrate the possible mechanism for OML delivery to induce CTLs.

DC-SIGN (CD209) is a type II membrane protein, which is now established as a mannose-binding protein [62], and which appears to be a major receptor for OMLs [63]. It exhibits higher avidity to mannose through multimer formation, while there is not a one-to-one correspondence between the mannose and carbohydrate recognition domains (CRD) of DC-SIGN because of the binding specificity for $\mathrm{N}$ acetylglucosamine (GlcNAc) and fructose (fuc) [62]. DCSIGN was initially reported as having specific binding for HIV gp120 [64], and further, ICAM-2 and ICAM-3 on T cells were identified as ligands [65]. Because the interaction between DC-SIGN and ICAM-3 is inhibited by added free mannose, mannose residues of ICAM-3 act as ligands for CRD of DC-SIGN [65]. Though the ligand binding has been considered to enhance $\mathrm{T}$ cell activation by MHC class II (and possibly MHC class I) molecules in a restricted manner $[66,67]$, the exact role of how DC-SIGN associates to induce and activate CTLs remains to be elucidated.

The initial immunological role of MMR/CD206 has been considered to be for the surveillance of invading pathogens such as Candida albicans and Pneumocystis carinii [68, 69]. MMR has eight CRD/C-type lectin-like carbohydrate recognition domains, one fibronectin type II repeat domain (Fn-II D), five CRDs (CRD4-8), which bind with mannose, but also fuc and GlcNAc [70, 71]. Targeted delivery using a specific antibody to MMR increases the uptake of delivered 
TABLE 1: Lectin-like receptors, complement receptors (CR), and ligands.

\begin{tabular}{lc}
\hline Receptors & Ligands \\
\hline DC-SIGN (CD209) & Fucose, mannose, N-acetylglucosamine \\
DEC-205(CD205) & Unknown \\
Mannose receptor (CD206) & Fucose, mannose, N-acetylglucosamine \\
CR1 (CD35) & C3b, iC3b \\
CR2 (CD21) & C3d \\
CR3 (CD11b/CD18, Mac-1) & iC3b \\
CR4 (CD11 c/CD18) & iC3b \\
CR5 (CRlg, VSIG4) & iC3b \\
\hline
\end{tabular}

antigens by phagocytic cells, resulting in the concomitant activation of both $\mathrm{CD}^{+}$and $\mathrm{CD}^{+}{ }^{+} \mathrm{T}$ cells through antigen presentation on MHC class I and class II molecules $[39,72]$. This observation suggests a possible role of MMR in the induction of CTLs when OML is used for immunization.

Though DEC-205/CD205 has 10 CRDs and is highly homologous with MMR, its avidity to any glycans has not been detected. It may be caused by the limited number of glycan structures to perform binding analysis [73-75]. DEC-205 expresses exclusively on mature DCs, but for macrophage and immature DCs, targeting DEC-205 to deliver liposome-containing vaccine antigen has the potential to improve the efficiency of CTL generation because of possible adjuvanticity [76].

To date, five members of CRs (CR1-5) have been identified, and all of which are associated with opsonophagocytosis through the activation of the complement system [77]. Expression of all CRs has been exclusively detected on monocyte/macrophage lineage cells, while the distinct presence of CR1 and CR2 on erythrocytes and B cells is known [78]. Table 1 shows the differential binding specificity of each CR for C3 fragments [79-81].

CRs appear generally to have other functions besides facilitating opsonophagocytosis, known as their classical role. CRIg/VSIG4 is a recently identified fifth member (CR5) of the CR family, the long form of which is identical to Z39Ig reported in earlier studies [78, 81]. CR5, CRIg/VSIG4/Z39Ig, on monocytic cells can bind $\mathrm{C} 3 \mathrm{~b}$ and $\mathrm{iC} 3 \mathrm{~b}$ to internalize opsonized materials in the same way as other CRs, while it appears to play other roles, such as B7 family molecules, to suppress the activation and proliferation of CTLs [82, 83]. In addition, CR2/CD21 on B cells was demonstrated to transduce positive signals for antibody production upon complex formation with CD19 and CD81 [84]. Taking into account that CRs have another function besides internalization, CR3 (CD11b/CD18, Mac-1) [85] and CR4 (CD11b) may play some role in generating CTLs upon taking up OML $[84,85]$.

\section{Possible Infectious Disease Targets for a Vaccine Strategy Using OMLs}

In order to prevent manifestation of some diseases whose main effectors are CTLs, administration of recombinant antigen as a vaccine requires particular adjuvants to induce
CTLs sufficiently to reject causative pathogens. To date, live vaccines are exclusively applied for universal prophylaxis of domestic animals, because they appear to have higher efficacy for illness given infection than either recombinant or inactivated vaccine containing adjuvant because of the advantage of induction efficiency of CTLs. Because vaccination with OMLs is expected to induce concomitant activation of both CTLs and Th1 cells, it has the potential to alter general approaches for infection control in domestic animals from live vaccines to recombinant antigen.

Based on increasing knowledge regarding OML-based vaccines, we have attempted to use them for control of some infectious diseases. Promising results using OMLs could be obtained for infection by N. caninum [10] and Leishmania major [53] using animal models. By administration of Neospora antigen NcGRA7 encased in OMLs to mice, NcGRA7-specific Th1 cells were generated, preventing the transition of the infection to the brain and transplacental vertical transmission [10]. Moreover, administration of apical membrane antigen 1 of Neospora using OMLs succeeded to reduce offspring mortality [11]. Whether the induced CTLs eliminated N. caninum in infected mice, it remained to determine increased numbers of the infection-specific CTLs by ELISpot assay, and estimate disease activity using genetically engineered protozoans expressing OVA as surrogate antigens. For the L. major infection, as earlier models with genetically engineered protozoans showed important roles of CTLs in the elimination of L. major [86] similar to the intracellular protozoan parasite Toxoplasma gondii [87], both antigen specific CTLs and Th1 cells induced by OML vaccination would show efficacy in preventing development of the illness.

We consider that CTL generation by OML-based vaccines can also be applied to Theileriosis. Theileriosis is a serious infection in cattle caused by tick-borne parasites, and is classified as a lymphoproliferative or hemoproliferative disease, depending on the principal pathogenic feature. $T$. parva and $T$. annulata are agents of lymphoproliferative Theileriosis [88, 89], and CTLs against T. annulata and T. parva are known to prevent disease progression [90, 91]. To date, live vaccine is known to be efficacious as a prophylactic agent. Administration of either T. annulata attenuated by long time in vitro culture or the infectionand-treatment method for T. parva $[92,93]$ are known as effective vaccination methods preventing the development of the lymphoproliferative disease. These results suggest that 
it is possible to use OML vaccines to prevent the onset of lymphoproliferative Theileriosis.

\section{Conclusion}

In accordance with the findings from many attempts to generate antigen-specific CTLs, this paper presents an overview of current trials concerning liposome-based vaccine delivery. We discuss the feasibility of using OML-based vaccine based on recently accumulated knowledge regarding carbohydrate recognition systems as targets for OML-based vaccine delivery systems. In order to practically use OML-based vaccine to introduce CTLs for prophylaxis of some infectious diseases, general use of ELISpot assays may be needed to monitor the efficacy of CTL generation. Taking into account the recent trend of surveying for latent infection of Mycobacterium tuberculosis [94] using this method, universal use of OMLbased vaccine to induce CTLs is not limited by this issue. Further studies characterizing the type of immune response induced by OML-based vaccine delivery in cattle are planned and should provide additional insights for the optimal development of OML-based vaccine to generate CTLs.

\section{Acknowledgments}

Introducing our works was supported by the Program for Promotion of Basic Research Activities for Innovative Biosciences (PROBRAIN) and the New Energy and Industrial Technology Development Organization (NEDO) of Japan. The cost of publication of this paper was defrayed in part by payment of page charges. This paper must therefore be marked as an advertisement in accordance with 18 U.S.C. Section 1734 solely to indicate this fact.

\section{References}

[1] Y. Ikehara, T. Niwa, L. Biao et al., "A carbohydrate recognitionbased drug delivery and controlled release system using intraperitoneal macrophages as a cellular vehicle," Cancer Research, vol. 66, no. 17, pp. 8740-8748, 2006.

[2] Y. Ikehara, N. Shiuchi, S. Kabata-Ikehara et al., "Effective induction of anti-tumor immune responses with oligomannose-coated liposome targeting to intraperitoneal phagocytic cells," Cancer Letters, vol. 260, no. 1-2, pp. 137-145, 2008.

[3] S. Sakaguchi, R. Setoguchi, H. Yagi, and T. Nomura, "Naturally arising Foxp3-expressing $\mathrm{CD} 25^{+} \mathrm{CD} 4^{+}$regulatory $\mathrm{T}$ cells in self-tolerance and autoimmune disease," Current Topics in Microbiology and Immunology, vol. 305, pp. 51-66, 2006.

[4] R.-F. Wang, G. Peng, and H. Y. Wang, "Regulatory T cells and Toll-like receptors in tumor immunity," Seminars in Immunology, vol. 18, no. 2, pp. 136-142, 2006.

[5] R. M. Zinkernagel, "Immunology taught by viruses," Science, vol. 271, no. 5246, pp. 173-178, 1996.

[6] A. M. Leen, C. M. Rooney, and A. E. Foster, "Improving T cell therapy for cancer," Annual Review of Immunology, vol. 25, pp. 243-265, 2007.

[7] S. A. Rosenberg, "Progress in the development of immunotherapy for the treatment of patients with cancer," Journal of Internal Medicine, vol. 250, no. 6, pp. 462-475, 2001.
[8] S. A. Rosenberg, J. C. Yang, and N. P. Restifo, "Cancer immunotherapy: moving beyond current vaccines," Nature Medicine, vol. 10, no. 9, pp. 909-915, 2004.

[9] N. Kojima, L. Biao, T. Nakayama, M. Ishii, Y. Ikehara, and K. Tsujimura, "Oligomannose-coated liposomes as a therapeutic antigen-delivery and an adjuvant vehicle for induction of in vivo tumor immunity," Journal of Controlled Release, vol. 129, no. 1, pp. 26-32, 2008.

[10] Y. Nishikawa, H. Zhang, Y. Ikehara, N. Kojima, X. Xuan, and N. Yokoyama, "Immunization with oligomannose-coated liposome-entrapped dense granule protein 7 protects dams and offspring from Neospora caninum infection in mice," Clinical and Vaccine Immunology, vol. 16, no. 6, pp. 792-797, 2009.

[11] H. Zhang, Y. Nishikawa, J. Yamagishi et al., "Neospora caninum: application of apical membrane antigen 1 encapsulated in the oligomannose-coated liposomes for reduction of offspring mortality from infection in BALB/c mice," Experimental Parasitology, vol. 125, no. 2, pp. 130-136, 2010.

[12] A. Hagiwara, T. Takahashi, K. Sawai et al., "Milky spots as the implantation site for malignant cells in peritoneal dissemination in mice," Cancer Research, vol. 53, no. 3, pp. 687-692, 1993.

[13] E. Mandache, E. Moldoveanu, and G. Savi, "The involvement of omentum and its milky spots in the dynamics of peritoneal macrophages," Morphologie et Embryologie, vol. 31, no. 2, pp. 137-142, 1985.

[14] H. F. J. Dullens, L. H. P. M. Rademakers, S. Cluistra et al., "Parathymic lymph nodes during growth and rejection of intraperitoneally inoculated tumor cells," Invasion and Metastasis, vol. 11, no. 4, pp. 216-226, 1991.

[15] C. Hodel, "Ultrastructural studies on the absorption of protein markers by the greater omentum," European Surgical Research, vol. 2, no. 6, pp. 435-449, 1970.

[16] L. Gattinoni, D. J. Powell Jr., S. A. Rosenberg, and N. P. Restifo, "Adoptive immunotherapy for cancer: building on success," Nature Reviews Immunology, vol. 6, no. 5, pp. 383-393, 2006.

[17] V. Racanelli and T. Manigold, "Presentation of HCV antigens to naive $\mathrm{CD} 8^{+} \mathrm{T}$ cells: why the where, when, what and how are important for virus control and infection outcome," Clinical Immunology, vol. 124, no. 1, pp. 5-12, 2007.

[18] C. R. Alving, V. Koulchin, G. M. Glenn, and M. Rao, "Liposomes as carriers of peptide antigens: induction of antibodies and cytotoxic $\mathrm{T}$ lymphocytes to conjugated and unconjugated peptides," Immunological Reviews, no. 145, pp. 5-31, 1995.

[19] G. Chikh and M.-P. Schutze-Redelmeir, "Liposomal delivery of CTL epitopes to dendritic cells," Bioscience Reports, vol. 22, no. 2, pp. 339-353, 2002.

[20] N. M. Wassef, C. R. Alving, and R. L. Richards, "Liposomes as carriers for vaccines," ImmunoMethods, vol. 4, no. 3, pp. 217222, 1994.

[21] M. Kovacsovics-Bankowski and K. L. Rock, "A phagosome-tocytosol pathway for exogenous antigens presented on MHC class I molecules," Science, vol. 267, no. 5195, pp. 243-246, 1995.

[22] C. R. Alving and N. M. Wassef, "Cytotoxic T lymphocytes induced by liposomal antigens: mechanisms of immunological presentation," AIDS Research and Human Retroviruses, vol. 10, no. 2, pp. S91-S94, 1994.

[23] G. G. Chikh, S. Kong, M. B. Bally, J.-C. Meunier, and M.-P. M. Schutze-Redelmeier, "Efficient delivery of Antennapedia homeodomain fused to CTL epitope with liposomes into dendritic cells results in the activation of CD8 ${ }^{+}$T cells," Journal of Immunology, vol. 167, no. 11, pp. 6462-6470, 2001. 
[24] M.-P. Schutze-Redelmeier, H. Gournier, F. Garcia-Pons et al., "Introduction of exogenous antigens into the MHC class I processing and presentation pathway by Drosophila antennapedia homeodomain primes cytotoxic T cells in vivo," Journal of Immunology, vol. 157, no. 2, pp. 650-655, 1996.

[25] K.-D. Lee, Y.-K. Oh, D. A. Portnoy, and J. A. Swanson, "Delivery of macromolecules into cytosol using liposomes containing hemolysin from Listeria monocytogenes," Journal of Biological Chemistry, vol. 271, no. 13, pp. 7249-7252, 1996.

[26] T. Nakanishi, A. Hayashi, J. Kunisawa et al., "Fusogenic liposomes efficiently deliver exogenous antigen through the cytoplasm into the MHC class I processing pathway," European Journal of Immunology, vol. 30, no. 6, pp. 1740-1747, 2000.

[27] H. Ishiwata, S. B. Sato, S. Kobayashi, M. Oku, A. VertutDoï, and K. Miyajima, "Poly(ethylene glycol) derivative of cholesterol reduces binding step of liposome uptake by murine macrophage-like cell line J774 and human hepatoma cell line HepG2," Chemical and Pharmaceutical Bulletin, vol. 46, no. 12, pp. 1907-1913, 1998.

[28] G. Chikh, M. Bally, and M.-P. Schutze-Redelmeier, "Characterization of hybrid CTL epitope delivery systems consisting of the Antennapedia homeodomain peptide vector formulated in liposomes," Journal of Immunological Methods, vol. 254, no. 1-2, pp. 119-135, 2001.

[29] D. D. Lasic, F. J. Martin, A. Gabizon, S. K. Huang, and D. Papahadjopoulos, "Sterically stabilized liposomes: a hypothesis on the molecular origin of the extended circulation times," Biochimica et Biophysica Acta, vol. 1070, no. 1, pp. 187-192, 1991.

[30] H. Ishiwata, A. Vertut-Doi, T. Hirose, and K. Miyajima, "Physical-chemistry characteristics and biodistribution of poly(ethylene glycol)-coated liposomes using poly(oxyethylene) cholesteryl ether," Chemical and Pharmaceutical Bulletin, vol. 43, no. 6, pp. 1005-1011, 1995.

[31] A. A. Gabizon, H. Shmeeda, and S. Zalipsky, "Pros and cons of the liposome platform in cancer drug targeting," Journal of Liposome Research, vol. 16, no. 3, pp. 175-183, 2006.

[32] R. Ignatius, K. Mahnke, M. Rivera et al., "Presentation of proteins encapsulated in sterically stabilized liposomes by dendritic cells initiates CD8 ${ }^{+}$T-cell responses in vivo," Blood, vol. 96, no. 10, pp. 3505-3513, 2000.

[33] J. W. Conlan, L. Krishnan, G. E. Willick, G. B. Patel, and G. D. Sprott, "Immunization of mice with lipopeptide antigens encapsulated in novel liposomes prepared from the polar lipids of various Archaeobacteria elicits rapid and prolonged specific protective immunity against infection with the facultative intracellular pathogen, Listeria monocytogenes," Vaccine, vol. 19, no. 25-26, pp. 3509-3517, 2001.

[34] L. Krishnan, S. Sad, G. B. Patel, and G. D. Sprott, "Archaeosomes induce long-term $\mathrm{CD}^{+}$cytotoxic $\mathrm{T}$ cell response to entrapped soluble protein by the exogenous cytosolic pathway, in the absence of CD4 $4^{+} \mathrm{T}$ cell help," Journal of Immunology, vol. 165, no. 9, pp. 5177-5185, 2000.

[35] L. Krishnan, S. Sad, G. B. Patel, and G. D. Sprott, "The potent adjuvant activity of archaeosomes correlates to the recruitment and activation of macrophages and dendritic cells in vivo," Journal of Immunology, vol. 166, no. 3, pp. 1885-1893, 2001.

[36] T. Nakanishi, J. Kunisawa, A. Hayashi et al., "Positively charged liposome functions as an efficient immunoadjuvant in inducing immune responses to soluble proteins," Biochemical and Biophysical Research Communications, vol. 240, no. 3, pp. 793-797, 1997.
[37] T. Nakanishi, J. Kunisawa, A. Hayashi et al., "Positively charged liposome functions as an efficient immunoadjuvant in inducing cell-mediated immune response to soluble proteins," Journal of Controlled Release, vol. 61, no. 1-2, pp. 233-240, 1999.

[38] B. Guy, N. Pascal, A. Françon et al., "Design, characterization and preclinical efficacy of a cationic lipid adjuvant for influenza split vaccine," Vaccine, vol. 19, no. 13-14, pp. 17941805, 2001.

[39] T. Keler, V. Ramakrishna, and M. W. Fanger, "Mannose receptor-targeted vaccines," Expert Opinion on Biological Therapy, vol. 4, no. 12, pp. 1953-1962, 2004.

[40] F. Sallusto, M. Cella, C. Danieli, and A. Lanzavecchia, "Dendritic cells use macropinocytosis and the mannose receptor to concentrate macromolecules in the major histocompatibility complex class II compartment: downregulation by cytokines and bacterial products," Journal of Experimental Medicine, vol. 182, no. 2, pp. 389-400, 1995.

[41] P. Machy, K. Serre, and L. Leserman, "Class I-restricted presentation of exogenous antigen acquired by Fc $\gamma$ receptormediated endocytosis is regulated in dendritic cells," European Journal of Immunology, vol. 30, no. 3, pp. 848-857, 2000.

[42] K. Serre, P. Machy, J.-C. Grivel et al., "Efficient presentation of multivalent antigens targeted to various cell surface molecules of dendritic cells and surface Ig of antigen-specific B cells," Journal of Immunology, vol. 161, no. 11, pp. 6059-6067, 1998.

[43] P. Guermonprez, N. Khelef, E. Blouin et al., "The adenylate cyclase toxin of Bordetella pertussis binds to target cells via the $\alpha \mathrm{M} \beta 2$ integrin (CD11b/CD18)," Journal of Experimental Medicine, vol. 193, no. 9, pp. 1035-1044, 2001.

[44] P. Guermonprez, C. Fayolle, M. J. Rojas, M. Rescigno, D. Ladant, and C. Leclerc, "In vivo receptor-mediated delivery of a recombinant invasive bacterial toxoid to $\mathrm{CD} 11 \mathrm{c}^{+} \mathrm{CD} 8 \alpha^{-} \mathrm{CD} 11 \mathrm{~b}^{\text {high }}$ dendritic cells," European Journal of Immunology, vol. 32, no. 11, pp. 3071-3081, 2002.

[45] M. L. Albert, J.-I. Kim, and R. B. Birge, " $\alpha(\mathrm{v}) \beta 5$ integrin recruits the CrkII-Dock180-Racl complex for phagocytosis of apoptotic cells," Nature Cell Biology, vol. 2, no. 12, pp. 899$905,2000$.

[46] Y. Ikehara and N. Kojima, "Development of a novel oligomannose-coated liposome-based anticancer drugdelivery system for intraperitoneal cancer," Current Opinion in Molecular Therapeutics, vol. 9, no. 1, pp. 53-61, 2007.

[47] E. W. Adams, D. M. Ratner, P. H. Seeberger, and N. Hacohen, "Carbohydrate-mediated targeting of antigen to dendritic cells leads to enhanced presentation of antigen to T cells," ChemBioChem, vol. 9, no. 2, pp. 294-303, 2008.

[48] J. S. Lam, H. Huang, and S. M. Levitz, "Effect of differential Nlinked and O-linked mannosylation on recognition of fungal antigens by dendritic cells," PLOS ONE, vol. 2, no. 10, article e1009, 2007.

[49] M. Luong, J. S. Lam, J. Chen, and S. M. Levitz, "Effects of fungal $\mathrm{N}$ - and O-linked mannosylation on the immunogenicity of model vaccines," Vaccine, vol. 25, no. 22, pp. 4340-4344, 2007.

[50] S. K. Singh, J. Stephani, M. Schaefer et al., "Targeting glycan modified OVA to murine DC-SIGN transgenic dendritic cells enhances MHC class I and II presentation," Molecular Immunology, vol. 47, no. 2-3, pp. 164-174, 2009.

[51] K.-C. Sheng, M. Kalkanidis, D. S. Pouniotis, M. D. Wright, G. A. Pietersz, and V. Apostolopoulos, "The adjuvanticity of a mannosylated antigen reveals TLR4 functionality essential for subset specialization and functional maturation of mouse dendritic cells," Journal of Immunology, vol. 181, no. 4, pp. 2455-2464, 2008. 
[52] T. Mizuochi, R. W. Loveless, A. M. Lawson et al., "A library of oligosaccharide probes (neoglycolipids) from $\mathrm{N}$-glycosylated proteins reveals that conglutinin binds to certain complextype as well as high mannose-type oligosaccharide chains," Journal of Biological Chemistry, vol. 264, no. 23, pp. 1383413839, 1989.

[53] Y. Shimizu, K. Yamakami, T. Gomi et al., "Protection against Leishmania major infection by oligomannose-coated liposomes," Bioorganic and Medicinal Chemistry, vol. 11, no. 7, pp. 1191-1195, 2003.

[54] B. Burke, "Macrophages as novel cellular vehicles for gene therapy," Expert Opinion on Biological Therapy, vol. 3, no. 6, pp. 919-924, 2003.

[55] S. R. Clarke, M. Barnden, C. Kurts, F. R. Carbone, J. F. Miller, and W. R. Heath, "Characterization of the ovalbumin-specific TCR transgenic line OT-I: MHC elements for positive and negative selection," Immunology and Cell Biology, vol. 78, no. 2, pp. 110-117, 2000.

[56] K. A. Hogquist, S. C. Jameson, W. R. Heath, J. L. Howard, M. J. Bevan, and F. R. Carbone, "T cell receptor antagonist peptides induce positive selection," Cell, vol. 76, no. 1, pp. 17-27, 1994.

[57] M. J. Barnden, J. Allison, W. R. Heath, and F. R. Carbone, "Defective TCR expression in transgenic mice constructed using cDNA-based $\alpha$ - and $\beta$-chain genes under the control of heterologous regulatory elements," Immunology and Cell Biology, vol. 76, no. 1, pp. 34-40, 1998.

[58] T. Ishida, H. Harashima, and H. Kiwada, "Liposome clearance," Bioscience Reports, vol. 22, no. 2, pp. 197-224, 2002.

[59] Y. Abe, Y. Kuroda, N. Kuboki, M. Matsushita, N. Yokoyama, and N. Kojima, "Contribution of complement component C3 and complement receptor type 3 to carbohydrate-dependent uptake of oligomannose-coated liposomes by peritoneal macrophages," Journal of Biochemistry, vol. 144, no. 5, pp. 563$570,2008$.

[60] M. W. Turner, "Mannose-binding lectin: the pluripotent molecule of the innate immune system," Immunology Today, vol. 17, no. 11, pp. 532-540, 1996.

[61] A. Avraméas, D. McIlroy, A. Hosmalin et al., "Expression of a mannose/fucose membrane lectin on human dendritic cells," European Journal of Immunology, vol. 26, no. 2, pp. 394-400, 1996.

[62] D. A. Mitchell, A. J. Fadden, and K. Drickamer, "A novel mechanism of carbohydrate recognition by the C-type lectins DC-SIGN and DC-SIGNR. Subunit organization and binding to multivalent ligands," Journal of Biological Chemistry, vol. 276, no. 31, pp. 28939-28945, 2001.

[63] H. Takagi, M. Numazaki, T. Kajiwara et al., "Cooperation of specific ICAM-3 grabbing nonintegrin-related 1 (SIGNR1) and complement receptor type 3 (CR3) in the uptake of oligomannose-coated liposomes by macrophages," Glycobiology, vol. 19, no. 3, pp. 258-266, 2009.

[64] B. M. Curtis, S. Scharnowske, and A. J. Watson, "Sequence and expression of a membrane-associated C-type lectin that exhibits CD4-independent binding of human immunodeficiency virus envelope glycoprotein gp120," Proceedings of the National Academy of Sciences of the United States of America, vol. 89, no. 17, pp. 8356-8360, 1992.

[65] T. B. H. Geijtenbeek, R. Torensma, S. J. van Vliet et al., "Identification of DC-SIGN, a novel dendritic cell-specific ICAM-3 receptor that supports primary immune responses," Cell, vol. 100, no. 5, pp. 575-585, 2000.

[66] A. Engering, T. B. H. Geijtenbeek, S. J. van Vliet et al., "The dendritic cell-specific adhesion receptor DC-SIGN internalizes antigen for presentation to T cells," Journal of Immunology, vol. 168 , no. 5 , pp. 2118-2126, 2002.
[67] P. J. Tacken, I. J. M. de Vries, K. Gijzen et al., "Effective induction of naive and recall $\mathrm{T}$-cell responses by targeting antigen to human dendritic cells via a humanized anti-DCSIGN antibody," Blood, vol. 106, no. 4, pp. 1278-1285, 2005.

[68] R. A. B. Ezekowitz, D. J. Williams, H. Koziel et al., "Uptake of Pneumocystis carinii mediated by the macrophage mannose receptor," Nature, vol. 351, no. 6322, pp. 155-158, 1991.

[69] L. Marodi, H. M. Korchak, and R. B. Johnston Jr., "Mechanisms of host defense against Candida species: I. Phagocytosis by monocytes and monocyte-derived macrophages," Journal of Immunology, vol. 146, no. 8, pp. 2783-2789, 1991.

[70] P. R. Taylor, S. Gordon, and L. Martinez-Pomares, “The mannose receptor: linking homeostasis and immunity through sugar recognition," Trends in Immunology, vol. 26, no. 2, pp. 104-110, 2005.

[71] M. E. Taylor, K. Bezouska, and K. Drickamer, "Contribution to ligand binding by multiple carbohydrate-recognition domains in the macrophage mannose receptor," Journal of Biological Chemistry, vol. 267, no. 3, pp. 1719-1726, 1992.

[72] V. Ramakrishna, J. F. Treml, L. Vitale et al., "Mannose receptor targeting of tumor antigen pmell7 to human dendritic cells directs anti-melanoma $\mathrm{T}$ cell responses via multiple HLA molecules," Journal of Immunology, vol. 172, no. 5, pp. 28452852, 2004.

[73] M. E. Taylor, J. T. Conary, M. R. Lennartz, P. D. Stahl, and K. Drickamer, "Primary structure of the mannose receptor contains multiple motifs resembling carbohydrate-recognition domains," Journal of Biological Chemistry, vol. 265, no. 21, pp. 12156-12162, 1990.

[74] H. Feinberg, S. Park-Snyder, A. R. Kolatkar, C. T. Heise, M. E. Taylor, and W. I. Weis, "Structure of a C-type carbohydrate recognition domain from the macrophage mannose receptor," Journal of Biological Chemistry, vol. 275, no. 28, pp. 2153921548, 2000

[75] W. Jian, W. J. Swiggard, C. Heufler et al., "The receptor DEC205 expressed by dendritic cells and thymic epithelial cells is involved in antigen processing," Nature, vol. 375, no. 6527, pp. 151-155, 1995.

[76] D. Hawiger, K. Inaba, Y. Dorsett et al., "Dendritic cells induce peripheral $\mathrm{T}$ cell unresponsiveness under steady state conditions in vivo," Journal of Experimental Medicine, vol. 194, no. 6, pp. 769-779, 2001.

[77] M. van Lookeren Campagne, C. Wiesmann, and E. J. Brown, "Macrophage complement receptors and pathogen clearance," Cellular Microbiology, vol. 9, no. 9, pp. 2095-2102, 2007.

[78] J. Q. He, C. Wiesmann, and M. van Lookeren Campagne, "A role of macrophage complement receptor CRIg in immune clearance and inflammation," Molecular immunology, vol. 45, no. 16, pp. 4041-4047, 2008.

[79] V. M. Holers, T. Kinoshita, and H. Molina, "The evolution of mouse and human complement C3-binding proteins: divergence of form but conservation of function," Immunology Today, vol. 13, no. 6, pp. 231-236, 1992.

[80] M. C. Carroll, "The complement system in regulation of adaptive immunity," Nature Immunology, vol. 5, no. 10, pp. 981-986, 2004.

[81] K. Y. Helmy, K. J. Katschke Jr., N. N. Gorgani et al., “CRIg: a macrophage complement receptor required for phagocytosis of circulating pathogens," Cell, vol. 124, no. 5, pp. 915-927, 2006.

[82] L. Vogt, N. Schmitz, M. O. Kurrer et al., "VSIG4, a B7 familyrelated protein, is a negative regulator of T cell activation," Journal of Clinical Investigation, vol. 116, no. 10, pp. 28172826, 2006. 
[83] X. Zang and J. P. Allison, “To be or not to be B7," Journal of Clinical Investigation, vol. 116, no. 10, pp. 2590-2593, 2006.

[84] F. R. Toapanta and T. M. Ross, "Complement-mediated activation of the adaptive immune responses: role of $\mathrm{C} 3 \mathrm{~d}$ in linking the innate and adaptive immunity," Immunologic Research, vol. 36, no. 1-3, pp. 197-210, 2006.

[85] M. R. W. Ehlers, "CR3: a general purpose adhesionrecognition receptor essential for innate immunity," Microbes and Infection, vol. 2, no. 3, pp. 289-294, 2000.

[86] S. Bertholet, A. Debrabant, F. Afrin et al., "Antigen requirements for efficient priming of $\mathrm{CD}^{+} \mathrm{T}$ cells by Leishmania major-infected dendritic cells," Infection and Immunity, vol. 73, no. 10, pp. 6620-6628, 2005.

[87] F. Dzierszinski, M. Pepper, J. S. Stumhofer et al., "Presentation of Toxoplasma gondii antigens via the endogenous major histocompatibility complex class I pathway in nonprofessional and professional antigen-presenting cells," Infection and Immunity, vol. 75, no. 11, pp. 5200-5209, 2007.

[88] D. J. Mckeever, "Theileria parva and the bovine CTL response: down but not out?" Parasite Immunology, vol. 28, no. 7, pp. 339-345, 2006.

[89] J. S. Ahmed, E. J. Glass, D. A. Salih, and U. Seitzer, "Innate immunity to tropical theileriosis," Innate Immunity, vol. 14, no. 1, pp. 5-12, 2008.

[90] G. Conze, J. D. M. Campbell, A. K. Nichani, E. J. Glass, R. L. Spooner, and J. S. Ahmed, "Evidence for strain specificity in cytotoxic T-lymphocyte-mediated, major histocompatibility complex class I-dependent killing of Theileria annulatainfected cells," Parasitology Research, vol. 84, no. 7, pp. 593595, 1998.

[91] W. I. Morrison, B. M. Goddeeris, A. J. Teale, et al., "Cytotoxic T-cells elicited in cattle challenged with Theileria parva (Muguga): evidence for restriction by class I MHC determinants and parasite strain specificity," Parasite Immunology, vol. 9, no. 5, pp. 563-578, 1987.

[92] G. Di Giulio, G. Lynen, S. Morzaria, C. Oura, and R. Bishop, "Live immunization against East Coast fever-current status," Trends in Parasitology, vol. 25, no. 2, pp. 85-92, 2009.

[93] U. Seitzer and J. Ahmed, "Tropical theileriosis: cytotoxic T lymphocyte response to vaccination," Vaccine, vol. 26, pp. G24-28, 2008.

[94] K. Ewer, J. Deeks, L. Alvarez et al., "Comparison of Tcell-based assay with tuberculin skin test for diagnosis of Mycobacterium tuberculosis infection in a school tuberculosis outbreak," The Lancet, vol. 361, no. 9364, pp. 1168-1173, 2003. 


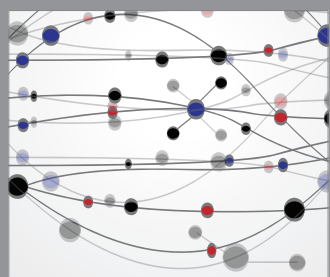

The Scientific World Journal
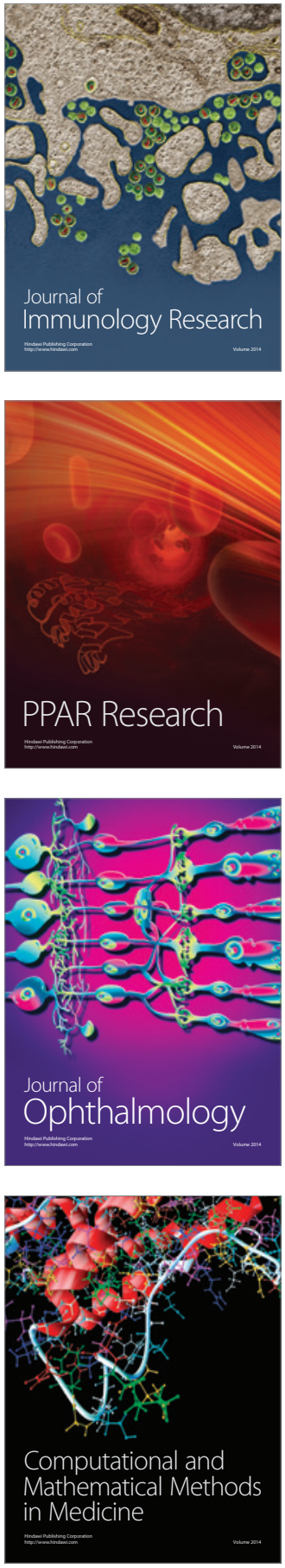

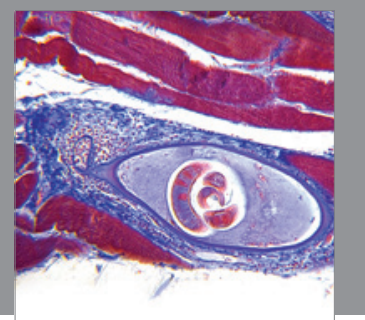

Gastroenterology

Research and Practice
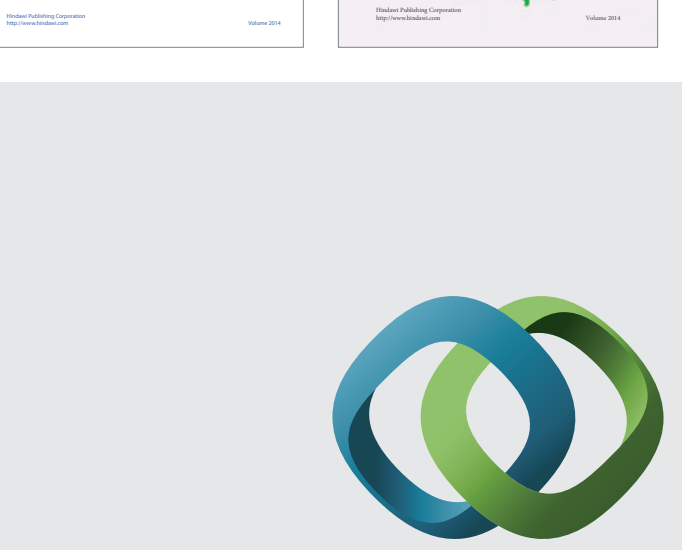

\section{Hindawi}

Submit your manuscripts at

http://www.hindawi.com
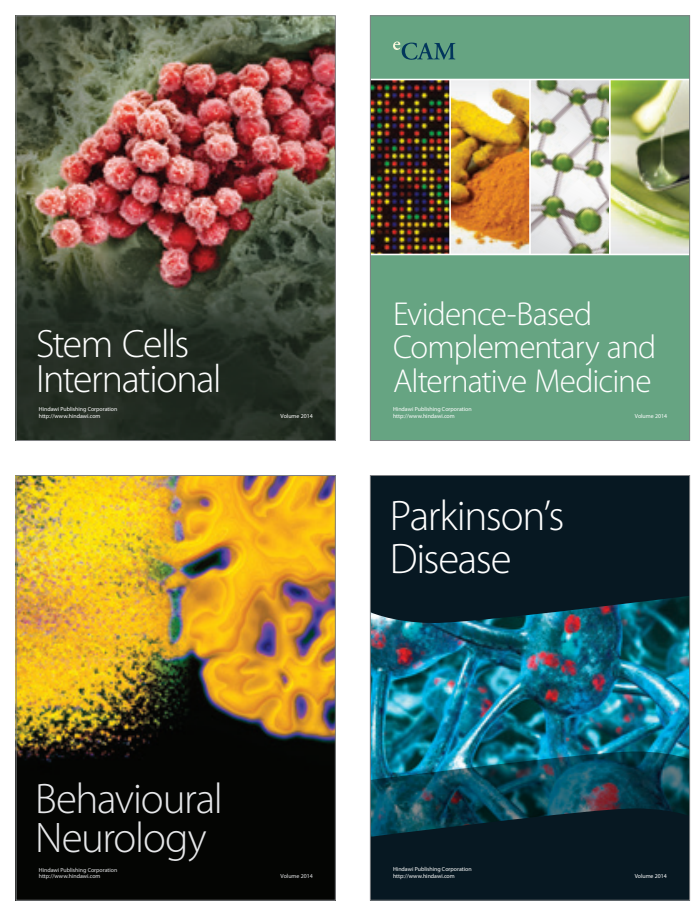

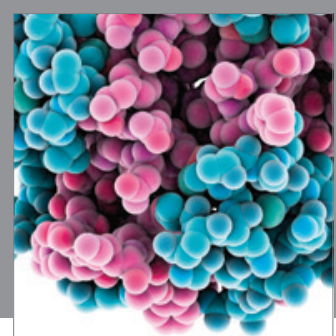

Journal of
Diabetes Research

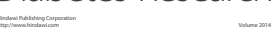

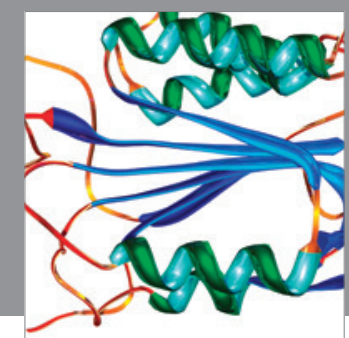

Disease Markers
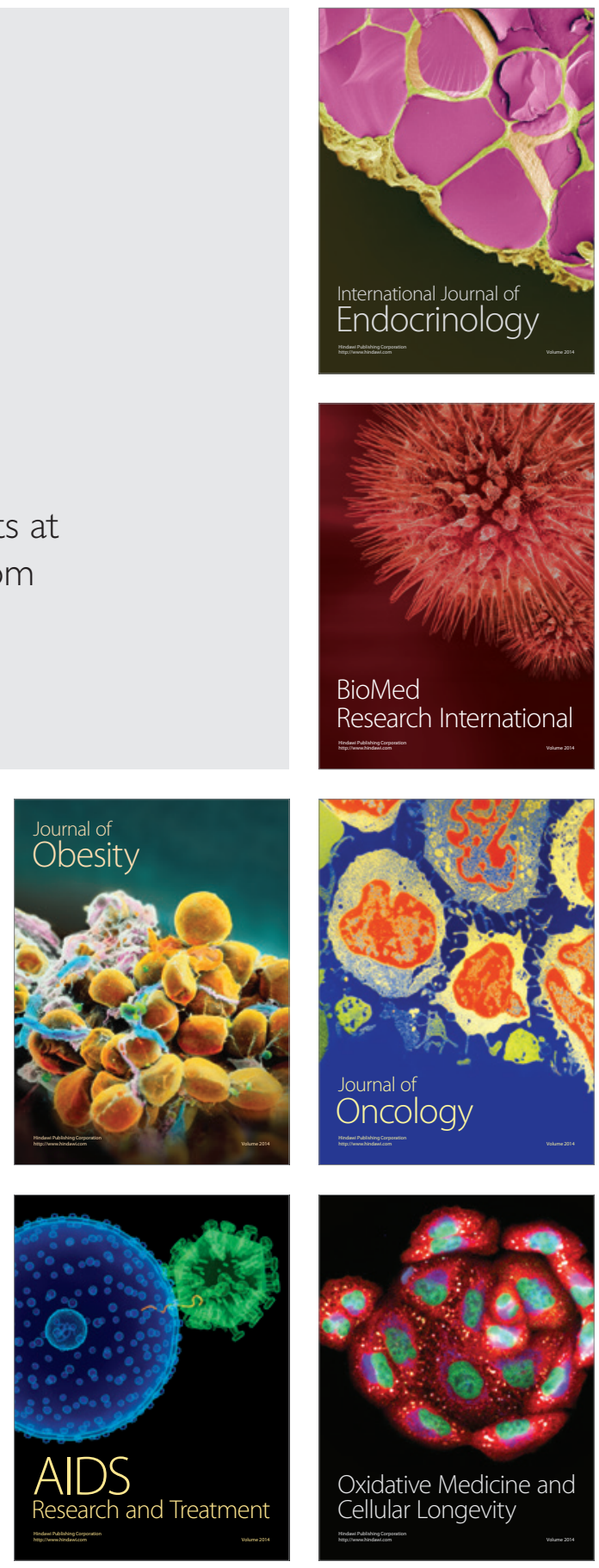